\title{
Polarized neutron powder diffraction studies of antiferromagnetic order in bulk and nanoparticle NiO
}

Brok, Erik; Lefmann, Kim; Deen, Pascale P.; Lebech, Bente; Jacobsen, Henrik; Nilsen, Gøran Jan; Keller, Lukas; Frandsen, Cathrine

Published in:

Physical Review B

Link to article, DOI:

10.1103/PhysRevB.91.014431

Publication date:

2015

Document Version

Publisher's PDF, also known as Version of record

Link back to DTU Orbit

Citation (APA):

Brok, E., Lefmann, K., Deen, P. P., Lebech, B., Jacobsen, H., Nilsen, G. J., Keller, L., \& Frandsen, C. (2015). Polarized neutron powder diffraction studies of antiferromagnetic order in bulk and nanoparticle NiO. Physical Review B, 91(1), 014431. https://doi.org/10.1103/PhysRevB.91.014431

\section{General rights}

Copyright and moral rights for the publications made accessible in the public portal are retained by the authors and/or other copyright owners and it is a condition of accessing publications that users recognise and abide by the legal requirements associated with these rights.

- Users may download and print one copy of any publication from the public portal for the purpose of private study or research.

- You may not further distribute the material or use it for any profit-making activity or commercial gain

- You may freely distribute the URL identifying the publication in the public portal 


\title{
Polarized neutron powder diffraction studies of antiferromagnetic order in bulk and nanoparticle $\mathrm{NiO}$
}

\author{
Erik Brok, ${ }^{1,2}$ Kim Lefmann, ${ }^{3}$ Pascale P. Deen, ${ }^{3,4}$ Bente Lebech, ${ }^{1,3}$ Henrik Jacobsen, ${ }^{3}$ Gøran Jan Nilsen, ${ }^{5}$ \\ Lukas Keller, ${ }^{6}$ and Cathrine Frandsen ${ }^{1}$ \\ ${ }^{1}$ Department of Physics, Technical University of Denmark, DK-2800 Kongens Lyngby, Denmark \\ ${ }^{2}$ Center for Electron Nanoscopy, Technical University of Denmark, DK-2800 Kongens Lyngby, Denmark \\ ${ }^{3}$ Nano-Science Center, Niels Bohr Institute, University of Copenhagen, DK-2100 Copenhagen Ø, Denmark \\ ${ }^{4}$ European Spallation Source AB, Lund, Sweden \\ ${ }^{5}$ Institut Max Von Laue Paul Langevin, F-38042 Grenoble, France \\ ${ }^{6}$ Laboratory for Neutron Scattering and Imaging, Paul Scherrer Institute, CH-5232 Villigen PSI, Switzerland
}

(Received 17 October 2014; revised manuscript received 23 December 2014; published 26 January 2015)

\begin{abstract}
In many materials it remains a challenge to reveal the nature of magnetic correlations, including antiferromagnetism and spin disorder. Revealing the spin structure in magnetic nanoparticles is further complicated by the large incoherent neutron scattering cross section from water adsorbed at the particle surfaces and by the broadening of diffraction peaks due to the finite crystallite size. Moreover, the spin structure in magnetic nanoparticles may deviate significantly from that of the corresponding bulk material because of the low-symmetry surroundings of surface atoms and the large relative surface contribution to the magnetic anisotropy. Here we explore the potential use of polarized neutron diffraction to reveal the magnetic structure in $\mathrm{NiO}$ bulk and nanoparticle powders by applying the $X Y Z$-polarization analysis method. Our investigations address in particular the spin orientation in bulk $\mathrm{NiO}$ and platelet-shaped $\mathrm{NiO}$ nanoparticles with thickness from greater than $200 \mathrm{~nm}$ down to $2.0 \mathrm{~nm}$. The advantage of the applied method is that it is able to clearly separate the structural, the magnetic, and the spin-incoherent scattering signals for all particle sizes. For platelet-shaped particles with thickness from greater than $200 \mathrm{~nm}$ down to $2.2 \mathrm{~nm}$ we find that the spin orientation deviates about $16^{\circ}$ from the primary (111) plane of the platelet-shaped particles. In the smallest particles $\left(2.0 \mathrm{~nm}\right.$ thick) we find the spins are oriented with a $30^{\circ}$ average angle to the primary (111) plane of the particles. The results show that polarization analyzed neutron powder diffraction is a viable method to investigate magnetic order in powders of antiferromagnetic nanoparticles.
\end{abstract}

DOI: 10.1103/PhysRevB.91.014431

PACS number(s): 75.25.-j, 75.50.Ee, 75.50.Tt, 61.05.fm

\section{INTRODUCTION}

Spin structures in systems where surface effects are important, such as nanoparticles or interfaces, are in general not well understood, although their magnetic properties may be interesting for use in technological applications [1]. Nanoparticles of antiferromagnetic materials do not possess a net magnetization of a useful size, but they still have application potential in combination with ferromagnetic or ferrimagnetic materials. In particular, exchange coupling to antiferromagnetic nanoparticles can greatly enhance the stability of the component that carries the net magnetization [2,3]. Before attempting to produce such a nanocomposite with technologically desired properties, it is important to understand the magnetic structure of the antiferromagnetic nanoparticles on its own.

Bulk NiO is antiferromagnetic below a Néel temperature of $523 \mathrm{~K}$ and was one of the first magnetic materials studied by neutron scattering in the pioneering work of Shull et al. [4]. These early neutron scattering experiments confirmed the existence of antiferromagnetic ordering as postulated by Néel [5]. The relatively high Néel temperature of $\mathrm{NiO}$ makes it a promising material for applications. Since the work of Shull et al. the magnetic structure of $\mathrm{NiO}$ has been studied by others [6-11] and there is now a consensus that the magnetic structure is antiferromagnetic with the antiferromagnetic modulation in one of the cubic $\langle 111\rangle$ directions $\left(\left\langle\frac{1}{2} \frac{1}{2} \frac{1}{2}\right\rangle\right.$-type modulation vector) and with the spins oriented close to the corresponding $\{111\}$ plane.
The spin orientation in $\mathrm{NiO}$ is difficult to determine, in particular for powdered samples, and in most investigations it is not determined with any appreciable accuracy. Using spherical neutron polarimetry, Ressouche et al. [11] determined the spin orientation in a single crystal of $\mathrm{NiO}$ to be within $1^{\circ}$ of the (111) plane perpendicular to the $\left[\frac{1}{2} \frac{1}{2} \frac{1}{2}\right]$ modulation vector.

The low-symmetry surroundings of surface atoms and the large surface contribution to the magnetic anisotropy mean that the spin structure in nanoparticles may be different from that of the bulk material $[12,13]$. This could also be the case for nanoparticles of $\mathrm{NiO}$, and the spin configuration at the surface of the $\mathrm{NiO}$ nanoparticles will certainly be of importance for their exchange coupling to other particles or surfaces.

The magnetic structure and in particular the spin orientation of (platelet-shaped) $\mathrm{NiO}$ nanoparticles have not been determined experimentally. In thin $\mathrm{NiO}$ films, a spin reorientation transition, in which the orientation of the $\mathrm{NiO}$ spin changes from being aligned within the film to being perpendicular to the film, is observed $[14,15]$. This transition depends not only on the thickness of the $\mathrm{NiO}$ film, but also on interaction with the support and on temperature. The spin orientation in thin $\mathrm{NiO}$ films may be influenced by epitaxial strain due to lattice mismatch between the film and its (MgO) support [14]. While there are important differences between $\mathrm{NiO}$ nanoparticles and thin $\mathrm{NiO}$ films, the two systems share the feature that a large proportion of the atoms reside on the surface and by this analogy it is interesting to investigate whether there is a spin reorientation in very thin platelet-shaped particles of $\mathrm{NiO}$. 
Neutron diffraction [16] is one of the few methods that can be used to determine the magnetic structure of antiferromagnetic materials. The conventional method of unpolarized neutron powder diffraction can be used on nanoparticle samples [17-19] and in fact Cooper et al. recently used this method on 7-nm NiO particles to compare the magnetic and nuclear correlation lengths, showing that a layer with spin disorder exists at the particle surfaces [19]. However, a very large spin-incoherent scattering contribution from water adsorbed on the surface of the particles and significant peak overlap because of finite-size broadening often severely hamper the analysis of neutron scattering data from nanoparticles. By using polarized neutrons and analyzing the spin state of the neutron before and after scattering, the magnetic part of the scattering can be separated from spin-incoherent and nuclear scattering. Removal of the spin-incoherent part of the scattering solves the problem of the large background and separation of the magnetic and nuclear signals solves the problem of overlapping magnetic and nuclear peaks. With the $X Y Z$-polarization analysis method the power of polarization analysis can be utilized on a multidetector instrument, making it possible to obtain separate magnetic and nuclear powder diffraction patterns [20].

Here we determine the spin orientation in platelet-shaped $\mathrm{NiO}$ nanoparticles using neutron diffraction with $X Y Z$ polarization analysis. The effect of particle size on the spin orientation is investigated for platelet-shaped $\mathrm{NiO}$ nanoparticles with thickness from greater than $200 \mathrm{~nm}$ down to $2.0 \mathrm{~nm}$. We find that the spin orientation in particles thicker than $2.2 \mathrm{~nm}$ is close to that expected for bulk NiO, whereas we see a significant spin reorientation in the particles that are only $2.0 \mathrm{~nm}$ thick.

\section{MAGNETIC STRUCTURE OF PLATELET-SHAPED NiO NANOPARTICLES}

\section{A. Magnetic structure of bulk NiO}

$\mathrm{NiO}$ crystallizes in the cubic $\mathrm{NaCl}$ structure $(F m \overline{3} m)$ with a room temperature lattice constant of $a=4.177 \AA$ [21]. At the Néel temperature of $T_{N}=523 \mathrm{~K}$ [6] the $\mathrm{Ni}^{2+}$ spins in $\mathrm{NiO}$ orders antiferromagnetically. Below $T_{N}$ there is a rhombohedral distortion of the lattice, corresponding to a slight contraction along one of the body diagonals of the cube. However, the departure of the rhombohedral angle from $90^{\circ}$ is very small, varying from 0 at $T_{N}$ to $0.075^{\circ}$ at $0 \mathrm{~K}$ (extrapolated) [22] and we will therefore continue to describe the system in terms of the more symmetric cubic unit cell. The antiferromagnetic modulation is along a body diagonal of the cube, $\langle 111\rangle$, and the spins are found to be aligned close to the corresponding $\{111\}$ planes $[7,8,10,11]$. A small in-plane anisotropy defines the easy axes of sublattice magnetization as the $\langle 11 \overline{2}\rangle$ directions [8-11]. The usual assumption is thus that each domain in bulk $\mathrm{NiO}$ consists of two sublattices, both with the spins oriented within a $\{111\}$ plane, but with the spins in one sublattice parallel to a $\langle 11 \overline{2}\rangle$ direction in this plane and the spins in the other sublattice antiparallel to it. The magnetic moment of the $\mathrm{Ni}^{2+}$ ion in $\mathrm{NiO}$ is often assumed to have the spin only value of $2 \mu_{B}$. However, magnetic $\mathrm{X}$-ray-scattering experiments [23,24] and density functional theory calculations [25] show a significant orbital contribution to the total magnetic moment and values between $1.8 \mu_{B}$ and $2.2 \mu_{B}$ have been reported [23-26] for the $\mathrm{Ni}^{2+}$ magnetic moment in $\mathrm{NiO}$.

\section{B. Platelet-shaped NiO nanoparticles}

High-purity platelet-shaped $\mathrm{NiO}$ nanoparticles with a reasonably narrow size distribution may be produced from thermal decomposition of platelet-shaped hexagonal $\mathrm{Ni}(\mathrm{OH})_{2}$ particles [27-31]. This results in particles of cubic $\mathrm{NiO}$ with $\{111\}$ planes as the platelet faces [31,32].

The magnetic properties of platelet-shaped $\mathrm{NiO}$ nanoparticles have been investigated with magnetization techniques [27-31,33-36], neutron scattering [17,37], and Mössbauer spectroscopy [17,29,31], revealing that the particles may undergo superparamagnetic relaxation and that they may carry a net magnetic moment. The net magnetic moment in antiferromagnetic nanoparticles is often attributed to uncompensated spins on the surface (or in the interior) of the particles and studies have found the magnetic moment of the particles to be roughly proportional to $N^{1 / 3}$ ( $N$ being the number of atomic spins in the particle), consistent with an uncompensated magnetic moment from random occupation of surface sites [31,38].

Other authors report an unexpected large uncompensated magnetic moment and explain this by a multisublattice model for the magnetic structure in $\mathrm{NiO}$ nanoparticles, where the simple model of two antiferromagnetic sublattices is replaced with one of two to eight sublattices depending on particle size [13,39,40]. However, Bahl et al. [31] demonstrate, based on magnetization measurements and Mössbauer spectroscopy studies, that it is not necessary to introduce a multisublattice model that is considerably more complicated than the twosublattice model usually assumed for bulk NiO.

Several authors have observed large coercivities and shifts of hysteresis loops (exchange bias) of $\mathrm{NiO}$ nanoparticles [28,33-36] and this is ascribed to exchange interaction between an antiferromagnetically ordered particle core and disordered spins at the particle surface that freeze in a spin-glass-like configuration at low temperatures depending on particle size.

The Néel temperature of the nanoparticles is found to be reduced with respect to the bulk value of $523 \mathrm{~K}$. For a sample of platelet-shaped $\mathrm{NiO}$ particles with a diameter of about 12 $\mathrm{nm}$ and thickness of $2 \mathrm{~nm}, T_{N} \approx 460 \mathrm{~K}$ was found using neutron diffraction [17]. A similar value can be expected for the nanoparticles to be investigated here.

Far infrared spectroscopy measurements on platelet-shaped $\mathrm{NiO}$ particles with average sizes of 43.5 and $5.7 \mathrm{~nm}$ showed magnetic resonance frequencies similar to observations in bulk $\mathrm{NiO}$, suggesting that the strong out-of-plane anisotropy, which makes it favorable for the spins to be oriented within the $\{111\}$ plane perpendicular to the direction of antiferromagnetic modulation, does not depend dramatically on particle size [41]. The weaker in-plane anisotropy, which makes it favorable for the spins to point in the $\langle 11 \overline{2}\rangle$ directions, on the other hand, may depend strongly on particle size as it has been shown by inelastic neutron scattering and Mössbauer spectroscopy mea- 
TABLE I. Allowed reflections for $\mathrm{NiO}$ with scattering vectors up to $4 \AA^{-1}$. The reflections with integer $h k l$ are nuclear $(\mathrm{N})$ and the half-integer-valued ones are magnetic (M). The multiplicity of a reflection is $j$. The magnetic reflections are calculated with [111] as the direction of antiferromagnetic modulation.

\begin{tabular}{lcccc}
\hline \hline$\{h k l\}$ & $q(\AA)^{-1}$ & $j$ & Allowed reflections & Type \\
\hline$\left\{\frac{1}{2} \frac{1}{2} \frac{1}{2}\right\}$ & 1.303 & 2 & $\left(\frac{1}{2} \frac{1}{2} \frac{1}{2}\right),\left(\frac{1}{2} \frac{1}{2} \frac{1}{2}\right)$ & $\mathrm{M}$ \\
$\left\{\frac{3}{2} \frac{1}{2} \frac{1}{2}\right\}$ & 2.495 & 6 & $\left(\frac{3}{2} \frac{1}{2} \frac{1}{2}\right),\left(\frac{1}{2} \frac{3}{2} \frac{1}{2}\right),\left(\frac{1}{2} \frac{1}{2} \frac{3}{2}\right),\left(\frac{3}{2} \frac{1}{2} \frac{1}{2}\right),\left(\frac{1}{2} \frac{3}{2} \frac{1}{2}\right),\left(\overline{\frac{1}{2}} \frac{1}{2} \frac{3}{2}\right)$ & $\mathrm{M}$ \\
$\{111\}$ & 2.605 & 8 & $(111),(\overline{1} 11),(1 \overline{1} 1),(11 \overline{1}),(\overline{111}),(1 \overline{11}),(\overline{1} 1 \overline{1}),(\overline{11} 1)$ & $\mathrm{N}$ \\
$\{200\}$ & 3.009 & 6 & $(200),(020),(002),(\overline{2} 00),(0 \overline{2} 0),(00 \overline{2})$ & $\mathrm{N}$ \\
$\left\{\frac{1}{2} \frac{3}{2} \frac{3}{2}\right\}$ & 3.278 & 6 & $\left(\frac{1}{2} \frac{3}{2} \frac{3}{2}\right),\left(\frac{3}{2} \frac{1}{2} \frac{3}{2}\right),\left(\frac{3}{2} \frac{3}{2} \frac{1}{2}\right),\left(\frac{1}{2} \frac{3}{2} \frac{3}{2}\right),\left(\frac{3}{2} \frac{1}{2} \frac{3}{2}\right),\left(\frac{3}{2} \frac{3}{2} \frac{1}{2}\right)$ & $\mathrm{M}$ \\
$\left\{\frac{3}{2} \frac{3}{2} \frac{3}{2}\right\}$ & 3.908 & 2 & $\left(\frac{3}{2} \frac{3}{2} \frac{3}{2}\right),\left(\frac{3}{2} \frac{3}{2} \frac{3}{2}\right)$ & $\mathrm{M}$ \\
\hline \hline
\end{tabular}

surements on platelet-shaped $\mathrm{NiO}$ particles with a thickness of $2.3 \mathrm{~nm}[37]$.

\section{NEUTRON POWDER DIFFRACTION}

The interpretation of our experimental results depends critically on the understanding of the cross sections for polarized neutron scattering. We will therefore briefly dwell on this topic by summarizing results relevant for our work.

\section{A. Structure factors}

In a neutron powder diffraction experiment the measured quantities are essentially the nuclear and magnetic structure factors of the crystal and magnetic structure of the sample as a function of the scattering vector $\mathbf{q}$. The nuclear structure factor is

$$
F_{N}(\mathbf{q})=\sum_{i} b_{i} \exp \left(i \mathbf{q} \cdot \boldsymbol{\Delta}_{i}\right)
$$

where $b_{i}$ is the scattering length of the atom (nucleus) at position $\boldsymbol{\Delta}_{i}$ in the unit cell and the sum is over all atoms in the unit cell. The magnetic structure factor is defined analogously to $F_{N}$ :

$$
F_{M}(\mathbf{q})=\sum_{i}\left\langle\mathbf{M}_{i, \perp}\right\rangle \exp \left(-i \mathbf{q} \cdot \boldsymbol{\Delta}_{i}\right)
$$

where $\mathbf{M}_{i, \perp}$ is the so-called magnetic interaction vector, which is the (microscopic) magnetization perpendicular to the scattering vector. The sum is over all magnetic moments in the unit cell. The magnetic unit cell of $\mathrm{NiO}$ is a cube with side length $2 a$ containing 32 nonmagnetic $\mathrm{O}^{2-}$ ions and 32 magnetic $\mathrm{Ni}^{2+}$ ions and it is most convenient to calculate both the nuclear and magnetic structure factors in terms of the larger magnetic cell [7]. With the Miller indices still referring to the original nuclear unit cell, the lowest $q$ nuclear reflections are the forms $\{111\}$ and $\{200\}$. The structure factor calculations show that the lowest $q$ allowed magnetic reflections are the $\left( \pm \frac{1}{2} \pm \frac{1}{2} \pm \frac{1}{2}\right)$ Friedel pair of the $\left\{\frac{1}{2} \frac{1}{2} \frac{1}{2}\right\}$ cubic form, i.e., only the two planes perpendicular to the [111] antiferromagnetic modulation direction contribute to the magnetic intensity at $q_{\left\{\frac{1}{2} \frac{1}{2} \frac{1}{2}\right\}}$. This has the consequence that the spin orientation can be uniquely determined from the intensity of the $\left\{\frac{1}{2} \frac{1}{2} \frac{1}{2}\right\}$ powder peak if the size of the $\mathrm{Ni}^{2+}$ magnetic moment is known. Equivalently, calculation of the structure factor for the $\left\{\frac{3}{2} \frac{1}{2} \frac{1}{2}\right\}$ form shows that only three of the 12 Friedel pairs in the $\left\{\frac{3}{2} \frac{1}{2} \frac{1}{2}\right\}$ cubic form contribute to the magnetic intensity at $q_{\left\{\frac{3}{2} \frac{1}{2} \frac{1}{2}\right\}}$. The allowed nuclear and magnetic reflections for scattering vectors lengths up to $4 \AA^{-1}$ and their multiplicities are given in Table I. The reduction of the multiplicities for the magnetic reflections is a consequence of the rhombohedral symmetry of the magnetic structure.

The structure factors for the first two nuclear reflections are

$$
\begin{aligned}
& F_{N,\{111\}}=32\left(b_{\mathrm{Ni}}-b_{\mathrm{O}}\right), \\
& F_{N,\{200\}}=32\left(b_{\mathrm{Ni}}+b_{\mathrm{O}}\right),
\end{aligned}
$$

where $b_{\mathrm{Ni}}=10.3(1) \mathrm{fm}$ and $b_{\mathrm{O}}=5.805(4) \mathrm{fm}$ are the (coherent) scattering lengths of $\mathrm{Ni}$ and $\mathrm{O}$, respectively. For all allowed magnetic reflections the magnetic structure factor is

$$
F_{M}(\mathbf{q})=32 M_{\perp}(\mathbf{q})
$$

\section{B. Obtaining the spin orientation from measured intensities}

While the periodic arrangement of the magnetic moments can be determined from the positions of the magnetic Bragg peaks, the intensity of the magnetic peaks can give information about the orientation of the spins with respect to the magnetic ordering vector. For a powder the elastic scattering cross section for nuclear scattering at a scattering vector length $q$ is (see, e.g., Ref. [16])

$$
\sigma_{N, \text { tot }, q}=\frac{N}{V_{0}} \frac{\lambda^{3}}{4 \sin \theta} \sum_{|\mathbf{q}|=q}\left|F_{N}(\mathbf{q})\right|^{2},
$$

where $N$ is the number of unit cells, $V_{0}$ is the unit cell volume, $2 \theta$ is the scattering angle, and $F_{N}(\mathbf{q})$ is the nuclear structure factor. The sum is over all reflections with a scattering vector of length $q$. When all reflections at a given $q$ have the same structure factor the sum can be replaced by $j\left|F_{N}(q)\right|^{2}$, where $j$ is the multiplicity of the reflection. The cross section in (5) is the scattering in the entire Debye-Scherrer cone and in an experiment it is necessary to correct for the finite coverage of the detector. The measured cross section on a detector of height $d$ at a distance $r$ from the sample is

$$
\sigma_{N, q}=\frac{d}{2 \pi r \sin (2 \theta)} \sigma_{N, \text { tot }, q} .
$$


The cross section for a reflection $h k l$ with scattering angle $2 \theta_{h k l}$ and multiplicity $j_{h k l}$ is then

$$
\sigma_{N, h k l}=\frac{d}{2 \pi r} \frac{N}{V_{0}} \frac{\lambda^{3}}{4 L_{\theta_{h k l}}} j_{h k l}\left|F_{N, h k l}\right|^{2},
$$

where $L_{\theta_{h k l}}=\sin \left(\theta_{h k l}\right) \sin \left(2 \theta_{h k l}\right)$ is sometimes referred to as the Lorentz factor. Similarly, the cross section for a magnetic $h k l$ reflection will be [16]

$$
\sigma_{M, h k l}=\frac{d}{2 \pi r} \frac{N}{V_{0}} \frac{\lambda^{3}}{4 L_{\theta_{h k l}}} j_{h k l}\left(\frac{\gamma r_{0}}{2}\right)^{2} g^{2} f^{2}(q)\left|F_{M, h k l}\right|^{2},
$$

where $\gamma=1.913$ is the gyromagnetic ratio, $r_{0}=2.8179 \mathrm{fm}$ is the classical electron radius, $g$ is the Landé $g$ factor, and $f(q)$ is the magnetic form factor.

For the $\left\{\frac{1}{2} \frac{1}{2} \frac{1}{2}\right\}$ reflection $M_{\perp}=M \sin (\alpha)$, where $\alpha$ is the angle between the (sublattice) spin direction and [111]. If the size of the magnetic moment is known, it is thus possible to obtain $\alpha$ from measurement of the $\left\{\frac{1}{2} \frac{1}{2} \frac{1}{2}\right\}$ cross section. In many experiments the cross section is not measured in absolute units and normalization is therefore necessary. If the normalization is with respect to a nuclear reflection, then $M_{\perp}=M \sin (\alpha)$ can be determined. However, from the relative intensities of two magnetic reflections it is possible to determine the spin orientation without knowing the size of the magnetic moment. In particular, we can obtain $\alpha$ by normalizing the $\left\{\frac{1}{2} \frac{1}{2} \frac{1}{2}\right\}$ intensity to that of the $\left\{\frac{3}{2} \frac{1}{2} \frac{1}{2}\right\}$ reflection.

The scattering vectors of the six allowed $\left\{\frac{3}{2} \frac{1}{2} \frac{1}{2}\right\}$ reflections all make an angle of $79.975^{\circ}$ ( or $180^{\circ}-79.975^{\circ}=100.025^{\circ}$ ) with [111]. The difference in the cross section of the individual magnetic reflections is contained in $M_{\perp}(\mathbf{q})$ and the ratio between the two measured cross sections will be

$$
\frac{\sigma_{M, \frac{1}{2} \frac{1}{2} \frac{1}{2}}}{\sigma_{M, \frac{3}{2} \frac{1}{2} \frac{1}{2}}}=\mathcal{L} \frac{2 \sin ^{2}(\alpha)}{\sum_{i=1}^{6} \sin ^{2}\left(\beta_{i}\right)},
$$

where the sum is over the six allowed reflections of the form $\left\{\frac{3}{2} \frac{1}{2} \frac{1}{2}\right\}, \beta_{i}$ is the angle between $\mathbf{M}(\mathbf{q})$ and the $i$ th of these scattering vectors, and

$$
\mathcal{L}=\frac{L_{\theta_{\left\{\frac{3}{2} \frac{1}{2} \frac{1}{2}\right\}}} f^{2}\left(q_{\left\{\frac{1}{2} \frac{1}{2} \frac{1}{2}\right\}}\right)}{L_{\theta_{\left\{\frac{1}{2} \frac{1}{2} \frac{1}{2}\right\}}} f^{2}\left(q_{\left\{\frac{3}{2} \frac{1}{2} \frac{1}{2}\right\}}\right)}
$$

If the magnetization in the (111) plane is assumed to be randomly distributed among the three $\langle 11 \overline{2}\rangle$ axes, the in-plane spin orientation can be averaged out, arriving at an expression for the sum in (9) that depends only on $\alpha$ :

$$
\begin{aligned}
\left\langle\sum_{i=1}^{6} \sin ^{2}\left(\beta_{i}\right)\right\rangle_{\delta}= & 2\left\{3-3 \cos ^{2}\left(79.975^{\circ}\right)+\sin ^{2}(\alpha)\right. \\
& \left.\times\left[3 \cos ^{2}\left(79.975^{\circ}\right)-\frac{3}{2} \sin ^{2}\left(79.975^{\circ}\right)\right]\right\},
\end{aligned}
$$

where \langle\rangle$_{\delta}$ denotes the average over the in-plane angle. Now $\alpha$ can be determined from the relative intensities of the first two magnetic peaks using Eq. (9). Using the same formalism, but with $79.975^{\circ}$ replaced by $48.527^{\circ}$ in $(11), \alpha$ can just as well be determined from the intensity of $\left\{\frac{1}{2} \frac{1}{2} \frac{1}{2}\right\}$ relative to $\left\{\frac{3}{2} \frac{3}{2} \frac{1}{2}\right\}$.

\section{IV. $X Y Z$-POLARIZATION ANALYSIS}

In the $X Y Z$-polarization analysis method the neutron beam is polarized and analyzed alternatingly along three orthogonal axes $(x, y$, and $z$ ). Using a spin flipper and a polarizing analyzer, scattering events that leave the spin of the neutron unchanged and scattering events that change the neutron spin state can be measured separately. These two types of measurements are commonly referred to as non-spin-flip (NSF) and spin-flip (SF) measurements. For each quantization axis the NSF and SF cross sections are measured, resulting in six measurements in total. This allows for a separation of nuclear, spin-incoherent, and magnetic scattering. With the $z$ axis vertical and the $x y$-plane in the horizontal scattering plane the magnetic cross section can be determined independently from the NSF and SF cross sections (see Refs. [20,42])

$$
\left(\frac{d \sigma}{d \Omega}\right)_{\mathrm{mag}}=2\left(\frac{d \sigma}{d \Omega}\right)_{\mathrm{SF}}^{x}+2\left(\frac{d \sigma}{d \Omega}\right)_{\mathrm{SF}}^{y}-4\left(\frac{d \sigma}{d \Omega}\right)_{\mathrm{SF}}^{z}
$$

and

$$
\left(\frac{d \sigma}{d \Omega}\right)_{\mathrm{mag}}=4\left(\frac{d \sigma}{d \Omega}\right)_{\mathrm{NSF}}^{z}-2\left(\frac{d \sigma}{d \Omega}\right)_{\mathrm{NSF}}^{x}-2\left(\frac{d \sigma}{d \Omega}\right)_{\mathrm{NSF}}^{y},
$$

where the superscripts refer to the quantization axis and the subscripts to the type of measurement (NSF or SF). The nuclear- and spin-incoherent cross sections can be determined similarly:

$$
\left(\frac{d \sigma}{d \Omega}\right)_{\mathrm{nuc}}=\frac{1}{6}\left[2\left(\frac{d \sigma}{d \Omega}\right)_{\mathrm{TNSF}}-\left(\frac{d \sigma}{d \Omega}\right)_{\mathrm{TSF}}\right]
$$

and

$$
\left(\frac{d \sigma}{d \Omega}\right)_{\mathrm{SI}}=\frac{1}{2}\left(\frac{d \sigma}{d \Omega}\right)_{\mathrm{TSF}}-\left(\frac{d \sigma}{d \Omega}\right)_{\mathrm{mag}},
$$

where TNSF (TSF) denotes the sum of all three NSF (SF) cross sections. If the sample is antiferromagnetic $\left(\frac{d \sigma}{d \Omega}\right)_{\mathrm{mag}}$ will be the differential cross section version of the total magnetic cross section in (8) and the measured $\left(\frac{d \sigma}{d \Omega}\right)_{\text {mag }}$ can be used to determine the spin orientation in $\mathrm{NiO}$ using (9). The $\left(\frac{d \sigma}{d \Omega}\right)_{\text {nuc }}$ will contain both the coherent nuclear cross section $(7)$, which gives rise to diffraction peaks, and isotope-incoherent scattering (mainly from the ${ }^{58} \mathrm{Ni}$ and ${ }^{60} \mathrm{Ni}$ isotopes), which should give rise to a flat background.

\section{EXPERIMENTAL DETAILS}

\section{A. Samples}

Four samples of platelet-shaped $\mathrm{NiO}$ nanoparticles were produced by thermal decomposition of platelet-shaped $\mathrm{Ni}(\mathrm{OH})_{2}$ nanoparticles in air. This method is known to produce platelet-shaped $\mathrm{NiO}$ particles with a thickness that depends on the annealing temperature (see, e.g., [27-31]). The $\mathrm{Ni}(\mathrm{OH})_{2}$ precursor is produced by chemical precipitation in aqueous solutions of $\mathrm{NaOH}$ and $\mathrm{Ni}\left(\mathrm{NO}_{3}\right)_{2} \cdot 6 \mathrm{H}_{2} \mathrm{O}$ as described in Ref. [28]. The four samples were annealed at $250^{\circ} \mathrm{C}, 300^{\circ} \mathrm{C}$, $350{ }^{\circ} \mathrm{C}$, and $600^{\circ} \mathrm{C}$ and the samples are named accordingly: $\mathrm{NiO} 250, \mathrm{NiO} 300, \mathrm{NiO} 350$, and $\mathrm{NiO} 600$. One sample of bulk $\mathrm{NiO}$ was produced by annealing at $1100{ }^{\circ} \mathrm{C}$ and another bulk 
sample was bought from Alfa Aesar (Puratronic ${ }^{\circledR}, 99.998 \%$ ). The two bulk samples are named $\mathrm{NiO} 1100$ and NiO-Alfa. All nanoparticle samples contained $2-3 \mathrm{~g}$ of $\mathrm{NiO}$ powder and the bulk samples contained 2.7 and $5 \mathrm{~g}$ for NiO1100 and NiO-Alfa, respectively. The bulk samples have a higher density than the nanoparticle samples and the same size of sample holder was used for NiO-Alfa and the nanoparticle samples. The samples were characterized with $\mathrm{x}$-ray powder diffraction using a Panalytic diffractometer with a $\mathrm{Cu}$ anode $(\lambda=1.54 \AA)$ and transmission electron microscope (TEM) images were recorded using a FEI Tecnai T20 microscope operated at $200 \mathrm{kV}$.

\section{B. Neutron diffraction experiments}

The spin orientation in the four nanoparticle samples and the NiO-Alfa bulk sample was studied at the D7 diffuse scattering spectrometer at Institut Laue-Langevin (ILL), Grenoble, France. The option for time-of-flight energy analysis using a Fermi chopper was not employed and the instrument was thus used as a powder diffractometer. The beam was polarized by a supermirror bender and detected by an array of 132 ${ }^{3} \mathrm{He}$ detectors giving a $q$ range of $0.28-3.85 \AA^{-1}$ with the $3.1-\AA$ neutrons selected by the highly oriented pyrolytic graphite (002) monochromator. A set of orthogonal ( $X Y Z)$ coils around the sample position makes it possible to define the spin direction for the incident neutrons and 66 supermirror benders are placed in front of the detector elements in order to analyze the spin state of the scattered neutrons. The analyzers are magnetized by a large number of $\mathrm{Nd}_{2} \mathrm{Fe}_{14} \mathrm{~B}$ magnets. A spin flipper of the Mezei type just before the $X Y Z$ coils makes it possible to reverse the polarization direction so that it is either parallel or antiparallel to the spin state being analyzed by the polarizing analyzers, thus enabling NSF and SF measurements. A guide field between the flipper and $X Y Z$ coils and between the $X Y Z$ coils and the analyzers ensures that the polarization of the neutrons is maintained. The sample was placed inside a standard orange cryostat and all measurements were performed at a temperature of $1.5 \mathrm{~K}$. To minimize spin-incoherent scattering the samples were dried in a vacuum oven at 0.1 bar for approximately $30 \mathrm{~min}$ at temperatures between $130^{\circ} \mathrm{C}$ and $150^{\circ} \mathrm{C}$ before and after they were placed in double-walled aluminium cans with outer diameters of 20 $\mathrm{mm}$ and inner diameters of 18 or $19 \mathrm{~mm}$.

Cadmium, empty can, and quartz measurements were performed to correct for background, absorption, and imperfect polarization. To correct for the detector efficiency and to obtain the cross sections on an absolute scale, a sample of vanadium powder was measured.

The experiment on D7 was inspired by previous attempts to determine the spin orientation in platelet-shaped $\mathrm{NiO}$ nanoparticles using unpolarized neutron powder diffraction at the DMC instrument at SINQ, the Paul Scherrer Institute, Switzerland. The experiments on DMC were performed with a neutron wavelength of $2.46 \AA$ provided by the PG002 monochromator. The samples were loaded in vanadium cylinders and kept at a temperature of $2 \mathrm{~K}$ by a standard orange cryostat during measurements. In the experiment at DMC both bulk samples and all nanoparticle samples were measured.
TABLE II. Average particle thickness $t_{\text {TEM }}$ determined from TEM images. The given uncertainty is the standard deviation of the mean. The width of the size distribution is approximately an order of magnitude larger. Also given is the volume weighted thickness $t_{\text {TEM }}^{V}$ and the average particle size, $d_{\mathrm{XRD}}$, determined with X-ray diffraction.

\begin{tabular}{lcccc}
\hline \hline Parameter & NiO250 & NiO300 & NiO350 & NiO600 \\
\hline$t_{\text {TEM }}(\mathrm{nm})$ & $1.99 \pm 0.09$ & $2.21 \pm 0.06$ & $3.29 \pm 0.10$ & \\
$t_{\text {TEM }}^{V}(\mathrm{~nm})$ & $2.5 \pm 0.6$ & $2.5 \pm 0.4$ & $3.7 \pm 0.5$ & \\
$d_{\text {XRD }}(\mathrm{nm})$ & $3.3 \pm 0.2$ & $4.9 \pm 0.6$ & $7.2 \pm 0.7$ & $28.3 \pm 0.2$ \\
\hline \hline
\end{tabular}

\section{RESULTS}

\section{A. Sample characterization}

X-ray powder diffraction (data not shown) confirms the conversion of the samples to cubic $F m \overline{3} m$ NiO with no visible sign of $\mathrm{Ni}(\mathrm{OH})_{2}$ or any other impurities. The average particle sizes of the nanoparticle samples $d_{\mathrm{XRD}}$ were determined from the broadening of the $\{111\}$ and $\{200\}$ peaks and are displayed in Table II. Because of the cubic symmetry the $\{111\}$ and $\{200\}$ reflections contain contributions from scattering vectors with many different orientations with respect to the particle shape and $d_{\mathrm{XRD}}$ is thus a measure of the average particle dimensions. With the available instrumental resolution none of the two bulk samples gave rise to any significant broadening of the diffraction peaks, indicating a crystallite size larger than 200 nm.

The particle size and morphology were further investigated with TEM. Example images are presented in Fig. 1. The images confirm that the particles retain the platelet shape

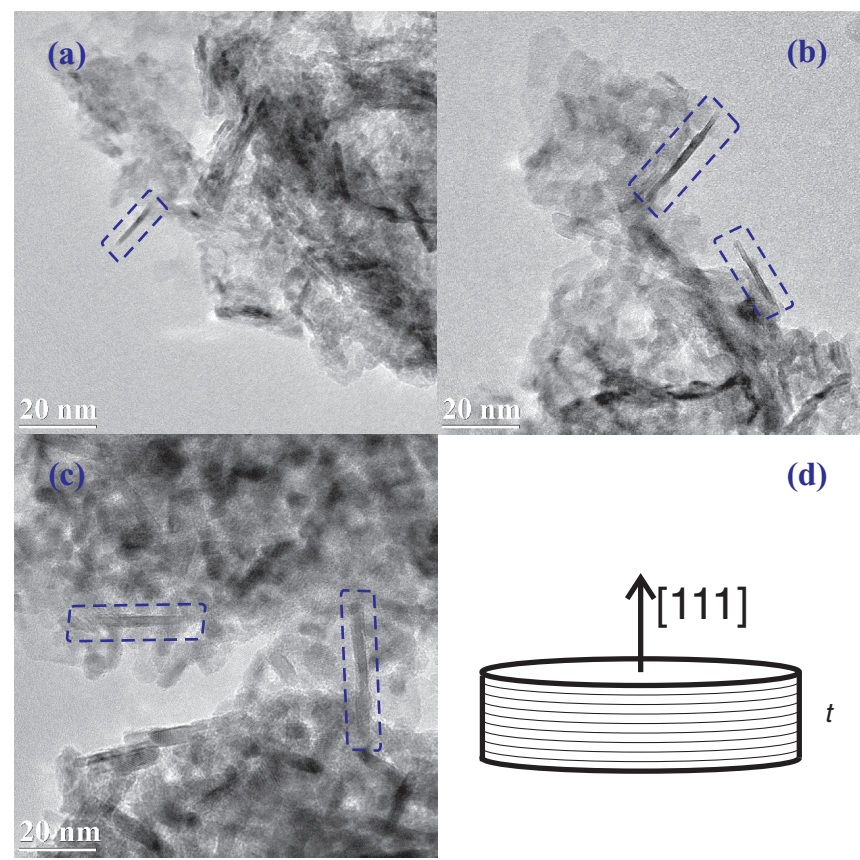

FIG. 1. TEM images of the samples (a) NiO250, (b) NiO300, and (c) NiO350. Particles seen edge on, like the ones highlighted in images (a)-(c), were used to measure the average particle thickness $t$. (d) Schematic drawing of a particle with the [111] direction normal to its face. 
of the precursor and the spacing between lattice fringes in high-resolution images is consistent with the $2.4-\AA d$ spacing of the $\{111\}$ planes of the cubic structure, confirming that the particle planes are parallel to the $\{111\}$ planes. The particles have a tendency to lie flat on the TEM grids and it is therefore difficult to measure the particle thickness. For the smaller particles ( $\mathrm{NiO} 250, \mathrm{NiO} 300$, and $\mathrm{NiO} 350)$ it was possible to find a number of particles that were standing upright. These particles are seen edge on and could therefore be used to estimate the average particle thickness. This was done by measuring approximately 40 particles for each of the samples $\mathrm{NiO} 250, \mathrm{NiO} 300$, and $\mathrm{NiO} 350$. For the $\mathrm{NiO} 600$ sample all particles were lying flat and it was not possible to determine the particle thickness. It should be noted that this method of determining the particle thickness may lead to an underestimate as the larger particles are less likely to stand upright than the smaller ones. The average particle thicknesses $t_{\text {TEM }}$ and the volume-weighted $\left(t^{3}\right.$-weighted) particle thickness $t_{\text {TEM }}^{V}$ determined with TEM are given in Table II together with $d_{\mathrm{XRD}}$ for the four nanoparticle samples.

\section{B. Unpolarized neutron powder diffraction}

The unpolarized neutron powder diffraction patterns from the four nanoparticle samples measured at DMC are displayed in Fig. 2. The expected nuclear and magnetic peaks from the $\mathrm{NiO}$ structure (see Table I) can be identified. At low $q$ there is a large signal that increases in strength with decreasing particle size. This signal falls off as $q^{-4}$ and is attributed to small-angle scattering from the particle surfaces. At larger $q$ the base level

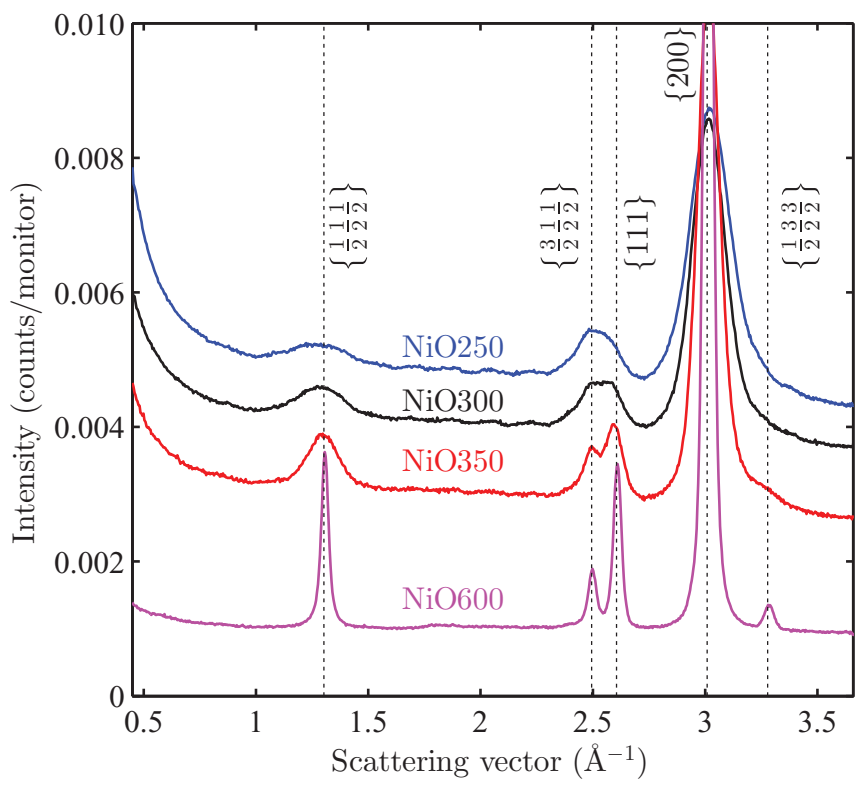

FIG. 2. (Color online) Unpolarized neutron powder diffraction data from platelet-shaped $\mathrm{NiO}$ nanoparticles measured at DMC. The measurements were performed at a temperature of $2 \mathrm{~K}$. The measurement times were about $12 \mathrm{~h}$ for the smallest particles (NiO250) and a few hours for the largest (NiO600). The scattering at low $q$ is small angle scattering from the surface of the particles. A constant value of 0.001 counts/monitor has been added to the $\mathrm{NiO} 300$ data to avoid overlap with other data. of the signal is, as we shall see in the following, primarily from spin-incoherent scattering. This large spin-incoherent signal can be attributed to water adsorbed on the particle surfaces and is therefore largest for the smallest particles, which have the largest relative surface area. The large background means that the signal-to-noise ratio is poor and therefore long counting times of up to $12 \mathrm{~h}$ were necessary to obtain data of a quality as in Fig. 2. The peak broadening is substantial and increases with decreasing particle size as expected. The broadening of the $\left\{\frac{1}{2} \frac{1}{2} \frac{1}{2}\right\}$ peak is particularly large.

In the diffraction patterns of the NiO600 sample and the two bulk samples (data not shown) the individual reflections can be clearly distinguished and the intensity of the individual peaks can be determined and used to calculate the spin angle by the procedure described in Sec. III B. From (9) the spin angle in $\mathrm{NiO} 600, \mathrm{NiO} 1100$, and $\mathrm{NiO}$-Alfa was determined to $\alpha=65.7^{\circ} \pm 0.3^{\circ}, \alpha=75.6^{\circ} \pm 0.3^{\circ}$, and $\alpha=76.3^{\circ} \pm 0.3^{\circ}$ respectively. Using these values of $\alpha$ the size of the $\mathrm{Ni}^{2+}$ moment can be determined from the relative intensities of the nuclear peaks and the $\left\{\frac{1}{2} \frac{1}{2} \frac{1}{2}\right\}$ peak obtaining $\mu=2.19 \mu_{B} \pm$ $0.07 \mu_{B}, \mu=2.01 \mu_{B} \pm 0.04 \mu_{B}$, and $\mu=2.02 \mu_{B} \pm 0.04 \mu_{B}$ for the three samples, respectively.

For the samples $\mathrm{NiO} 250, \mathrm{NiO} 300$, and $\mathrm{NiO} 350$, which consist of smaller particles, the peak broadening gives rise to significant peak overlap and it is very difficult to obtain the intensities of the two individual reflections, which are needed to calculate $\alpha$. Even determination of the intensity of the principal magnetic peak is problematic for the smallest particles, because the peak is very broad and overlaps with the strong signal at low $q$. These difficulties prevent accurate determination of the spin orientation, in the three samples with smallest particle thicknesses, from the unpolarized data. To solve these problems we turned to the technique of $X Y Z$-polarization analysis.

\section{Polarized neutron powder diffraction}

\section{Separation of scattering contributions}

The data from the $X Y Z$-polarization analysis experiment at D7 is presented in Fig. 3. Figures 3(a)-3(e) show the separated nuclear, magnetic, and spin-incoherent cross sections for the bulk and nanoparticle samples. The separation of the three scattering contributions works well and the statistics are good despite the very large spin-incoherent scattering signal from the nanoparticle samples. The dips in the spin-incoherent cross section at the positions of the nuclear Bragg peaks are artifacts of the correction for the imperfect polarization. No depolarization of the beam by the sample was observed. The magnetic Bragg reflections in $\left(\frac{d \sigma}{d \Omega}\right)_{\mathrm{mag}}$ are well resolved and the corresponding background is almost zero. The baseline of the nuclear signal has a value close to $0.41 \mathrm{~b} / \mathrm{sr}$ as expected for nuclear isotope incoherent scattering from $\mathrm{Ni}$. There is, however, an increase in the nuclear background for decreasing particle size, most likely due to structural disorder in the nanoparticles. The successful separation of the three scattering contributions is confirmed by the similarity of SF and NSF magnetic cross sections (not shown), which should be equivalent for a randomly oriented powder of particles with collinear antiferromagnetic order [20]. During the experiment the counting rates in the six scattering channels 

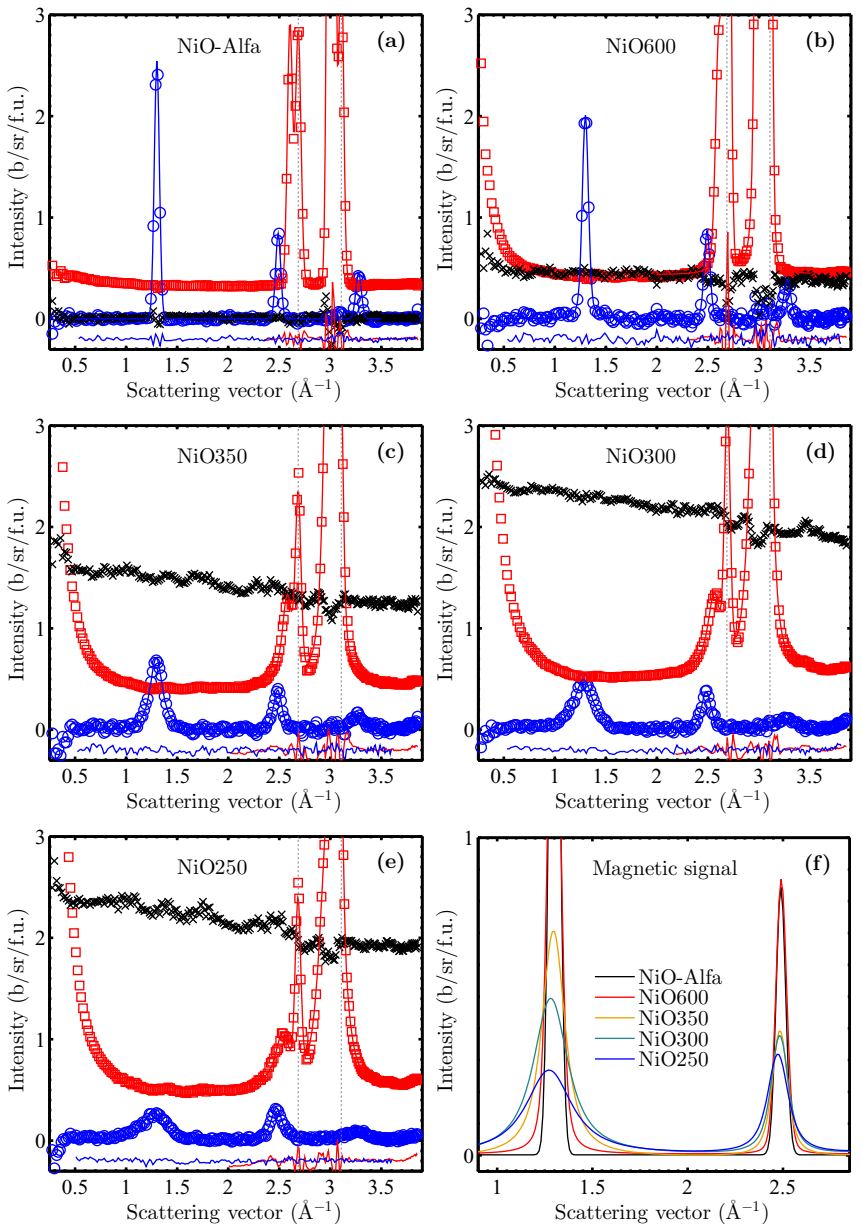

FIG. 3. (Color online) Polarization analyzed neutron powder diffraction patterns of (a) the bulk NiO-Alfa sample and (b)-(e) the four samples of platelet-shaped $\mathrm{NiO}$ nanoparticles. The separated magnetic (o), nuclear $(\square)$, and spin-incoherent $(\times)$ cross sections are shown. The full lines are the fits explained in the text. (f) Fits to the $\left\{\frac{1}{2} \frac{1}{2} \frac{1}{2}\right\}$ and $\left\{\frac{3}{2} \frac{1}{2} \frac{1}{2}\right\}$ magnetic peaks shown for all samples [notice that the intensity scale is changed with respect to (a)-(e)]. The blue and red lines below the data in (a)-(e) show the difference between model and data for the magnetic and nuclear scattering.

were compared, showing no difference between the three polarization directions. This means that there is no significant degree of preferred orientation in the powder.

The samples were measured in aluminum cans and therefore the $\mathrm{Al}\{111\}$ and $\{200\}$ reflections are seen in the nuclear diffraction pattern together with the expected peaks from the $\mathrm{NiO}$ structure. The overlap between the $\mathrm{NiO}$ and $\mathrm{Al}$ peaks in the nuclear signal makes the intensities of the individual nuclear peaks difficult to determine accurately.

The magnetic signal, however, is well separated from the nuclear Bragg peaks and from the strong signal at low $q$, which goes in the nuclear channel as expected. The magnetic part of the diffraction patterns of the five measured samples is shown in Fig. 3(f). The magnetic diffraction signal from the bulk sample is fitted with Gaussian line shapes, whereas Voigt line shapes are used for the nanoparticle data. In the bulk data the Gaussian widths are determined by the instrumental resolution. In the Voigt fits of the nanoparticle data the Gaussian widths are
TABLE III. Comparison of the magnetic correlation length in the [111] direction determined from the width of the $\left\{\frac{1}{2} \frac{1}{2} \frac{1}{2}\right\}$ peak with the particle thickness determined with TEM. The particle thickness at an angle of $70.5^{\circ}$ to the surface normal, corresponding to the other $\langle 111\rangle$ directions, is also given for comparison.

\begin{tabular}{lcccc}
\hline \hline Parameter & $\mathrm{NiO} 250$ & $\mathrm{NiO} 300$ & $\mathrm{NiO} 350$ & NiO600 \\
\hline$\xi_{[111], \mathrm{NPD}}(\mathrm{nm})$ & $2.26 \pm 0.08$ & $3.11 \pm 0.07$ & $5.3 \pm 0.2$ & $28 \pm 2$ \\
$t_{\mathrm{TEM}}^{V}(\mathrm{~nm})$ & $2.5 \pm 0.6$ & $2.5 \pm 0.4$ & $3.7 \pm 0.5$ & \\
$\frac{t_{\mathrm{TEM}}^{V}}{\cos \left(70.5^{\circ}\right)}(\mathrm{nm})$ & $8 \pm 2$ & $7.4 \pm 1.2$ & $11 \pm 2$ & \\
\hline \hline
\end{tabular}

fixed to the values obtained from the bulk fit. The Lorentzian widths are free parameters in the fits and are assumed to be due to the finite crystallite (particle) size.

\section{Direction of antiferromagnetic modulation}

The magnetic structure factor of the $\left\{\frac{1}{2} \frac{1}{2} \frac{1}{2}\right\}$ reflection (see Table I) is nonzero only for the reflections corresponding to planes perpendicular to the antiferromagnetic modulation direction (the [111] direction). This means that the broadening of the $\left\{\frac{1}{2} \frac{1}{2} \frac{1}{2}\right\}$ peak gives the magnetic correlation length in the [111] direction. The magnetic correlation length was determined from the full width at half maximum of the Lorentzian component of the Voigt fit. This correlation length $\xi_{[111], N P D}$ is given in Table III for the four nanoparticle samples together with the volume-weighted particle thickness $t_{\text {TEM }}^{V}$ already given in Table II. Here $\xi_{[111], N P D}$ is compared to the particle thickness determined with TEM rather than the $\mathrm{X}$-ray particle size, which is an average over many directions with respect to the particle shape. The sizes of $\xi_{[111], N P D}$ and $t_{\text {TEM }}^{V}$ are similar. The [111] direction makes an angle of about $70.5^{\circ}$ with the other $\langle 111\rangle$-type directions and consequently the particle size in any of the $\langle 111\rangle$ directions that are not normal to the particle plane is $t_{\mathrm{TEM}}^{V} / \cos \left(70.5^{\circ}\right)=3.00 t_{\mathrm{TEM}}^{V}$. This size is also given in Table II and is 2.1-3.5 times larger than $\xi_{[111], N P D}$. This leads us to conclude that the direction of antiferromagnetic modulation is typically perpendicular to the particle plane. We cannot, however, determine with certainty if the difference between $\xi_{[111], \mathrm{NPD}}$ and $t_{\mathrm{TEM}}^{V}$, in particular for the NiO350 sample, is due to an inaccurate estimate of the particle thickness with TEM or is because the antiferromagnetic modulation direction in some particles is not the [111] direction perpendicular to the particle plane.

\section{Spin orientation}

As discussed in Sec. III B, the spin angle $\alpha$ with respect to the scattering vector can be determined from the relative intensity of the first two magnetic peaks without any assumptions about the size of the magnetic moment. In the previous section we concluded that the direction of antiferromagnetic modulation is perpendicular to the plane of the particle. The particle shape thus imposes a reduced symmetry on the magnetic structure. This reduced symmetry means that $\alpha$ is not only the angle between the spin and the scattering vector, but also the angle between the spin and the normal to the particle plane. Figure 4 shows $\alpha$ for the four nanoparticle samples and the NiO-Alfa bulk sample. The intensities have 


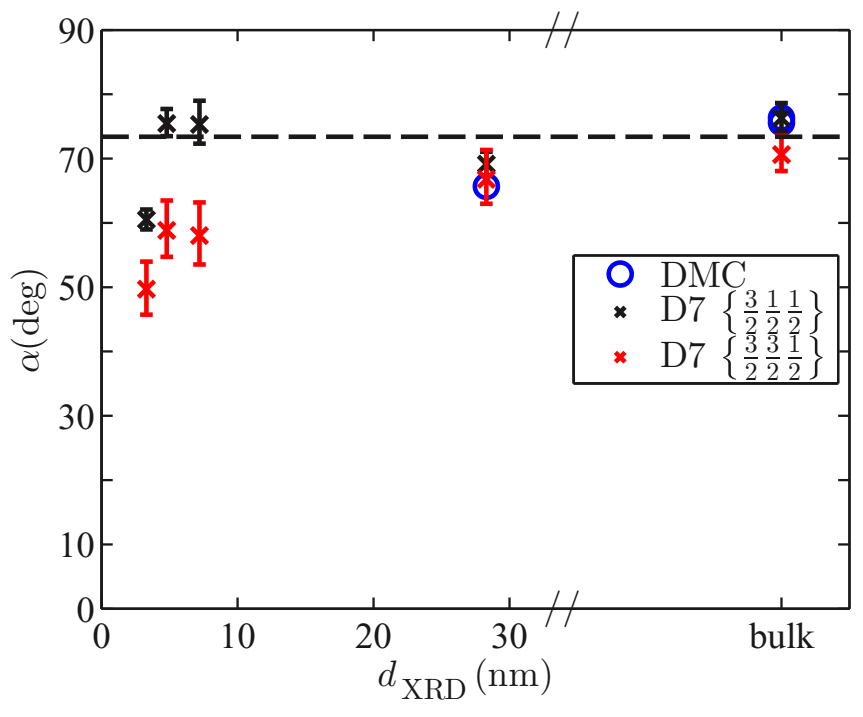

FIG. 4. (Color online) Spin angle with respect to the [111] direction normal to the particle plane calculated from the measured intensities of the magnetic reflections using Eq. (9). The calculated value of $\alpha$ is plotted against the $\mathrm{x}$-ray particle size, except for the bulk sample, which has a crystallite size larger than $200 \mathrm{~nm}$ and is arbitrarily placed on the axis. The black and red crosses are the results from the polarized experiment at D7 using $\left\{\frac{3}{2} \frac{1}{2} \frac{1}{2}\right\}$ and $\left\{\frac{3}{2} \frac{3}{2} \frac{1}{2}\right\}$ for normalization, respectively, and the blue circles are the results from the unpolarized experiment at DMC. The error bars on the DMC data points are considerably smaller than the diameter of the circles. The dashed line is the average spin angle of $74^{\circ}$ for $\mathrm{NiO} 300$, $\mathrm{NiO} 350, \mathrm{NiO600}$, and the bulk samples determined from normalizing with respect to $\left\{\frac{3}{2} \frac{1}{2} \frac{1}{2}\right\}$.

been determined by integration of the Voigt fits and the uncertainties have been estimated from the uncertainties on the fit parameters using a Monte Carlo approach.

From the relative intensities of the $\left\{\frac{1}{2} \frac{1}{2} \frac{1}{2}\right\}$ and $\left\{\frac{3}{2} \frac{1}{2} \frac{1}{2}\right\}$ reflections a spin angle of $\alpha \approx 74^{\circ}$ was found for all samples except $\mathrm{NiO} 250$, corresponding to an out-of-plane spin angle of about $16^{\circ}$. For $\mathrm{NiO} 250$, which is the sample with the smallest particle size $\left(t_{\mathrm{TEM}}=1.99 \pm 0.09 \mathrm{~nm}\right), \alpha \approx 60^{\circ}$ corresponding to $30^{\circ}$ out of the plane is found. The spin angle was also determined from the relative intensities of the $\left\{\frac{1}{2} \frac{1}{2} \frac{1}{2}\right\}$ and $\left\{\frac{3}{2} \frac{3}{2} \frac{1}{2}\right\}$ reflections. These results agree well with the ones obtained by normalizing to $\left\{\frac{3}{2} \frac{1}{2} \frac{1}{2}\right\}$ for the NiO-Alfa and NiO600 samples. For the smaller particles sizes, however, the normalization to $\left\{\frac{3}{2} \frac{3}{2} \frac{1}{2}\right\}$ results in a smaller $\alpha$, i.e., a larger out-of-plane spin component (cf. Fig. 4). The spin angles determined from the unpolarized data for the two bulk samples and NiO600 in Sec. VIB are also plotted in Fig. 4, showing excellent agreement with the results from the polarized experiment.

The intensities of the nuclear peaks in the polarized data could not be determined accurately because of the overlapping aluminium peaks and consequently the size of the magnetic moment could not be established in the same way as was done for the two bulk samples and NiO600 from the unpolarized data. Instead we normalized the intensities of the $\left\{\frac{1}{2} \frac{1}{2} \frac{1}{2}\right\}$ reflection of each nanoparticle sample to that of the NiO-Alfa sample, for which $\mu$ had already been determined from the unpolarized measurements, obtaining magnetic moments of
$2.20 \mu_{B} \pm 0.05 \mu_{B}, 1.98 \mu_{B} \pm 0.06 \mu_{B}, 2.08 \mu_{B} \pm 0.05 \mu_{B}$, and $1.97 \mu_{B} \pm 0.08 \mu_{B}$ for samples $\mathrm{NiO} 600, \mathrm{NiO} 350, \mathrm{NiO} 300$, and $\mathrm{NiO} 250$, respectively.

\section{DISCUSSION}

We observe a large broadening of the $\left\{\frac{1}{2} \frac{1}{2} \frac{1}{2}\right\}$ peak in the nanoparticle samples. This means that the magnetic correlation length in the direction of antiferromagnetic modulation is shorter than in the directions corresponding to the other magnetic peaks, indicating that the direction of antiferromagnetic modulation is not independent of the shape of the particles. For the samples with average particle thickness up to $3.7 \mathrm{~nm}$ (samples $\mathrm{NiO} 250, \mathrm{NiO} 300$, and $\mathrm{NiO} 350$ ) the magnetic correlation length in the [111] direction, $\xi_{[111], N P D}$ was similar to the volume-weighted particle thickness $t_{\text {TEM }}^{V}$ and significantly smaller than the particle size in the other $\langle 111\rangle$ directions. This strongly indicates that the direction of antiferromagnetic modulation is perpendicular to the plane of the platelet-shaped particles. The fact that $\xi_{[111], N P D}$ is somewhat larger than $t_{\text {TEM }}^{V}$ can be explained by an underestimate of the particle size from TEM as discussed in Sec. VIA. The magnetic correlation length would also be larger than the particle size determined with TEM if the magnetic (and crystallographic) order continues from one particle to the next. This may happen for epitaxially aligned particles [43]. As discussed below, we see no overall preferred orientation of the particles in the sample.

In principle, the similarity of the magnetic correlation length to the particle thickness and its dissimilarity to the particle dimensions in the other $\langle 111\rangle$ directions could be explained by an antiferromagnetic modulation direction along one of $\langle 111\rangle$ directions that are not perpendicular to the particle plane if there was a thick layer of disordered spins at the particle surfaces. This model, however, would not explain why the $\left\{\frac{1}{2} \frac{1}{2} \frac{1}{2}\right\}$ peak is significantly broader than the other magnetic peaks and we must therefore discard it.

For the NiO600 sample it was not possible to determine the particle thickness from TEM images, but given the platelet shape of the particles it seems likely that there is a similar relationship between the symmetry of the particle shape and the antiferromagnetic order in this sample. The particles in the bulk $\mathrm{NiO}$ samples are not expected to consist of a single magnetic domain and the discussion about the magnetic structure with respect to the particle shape is therefore less relevant.

It is interesting, but perhaps not so surprising, that the shape of the particle induces a reduced symmetry on the magnetic structure. The reduced symmetry of the magnetic structure is probably related by exchange striction to the phase transition from a cubic to a rhombohedral structure at $T_{N}$ and in turn related to the conversion of platelet-shaped hexagonal $\mathrm{Ni}(\mathrm{OH})_{2}$ particles to platelet-shaped rhombohedral $\mathrm{NiO}$ particles with the rhombohedral axis normal to the particle plane.

In bulk $\mathrm{NiO}$ the spin orientation is usually assumed to be within the $\{111\}$ plane perpendicular to the antiferromagnetic modulation direction $[10,11]$ and it is thus surprising that we find an out-of-plane spin angle of $14^{\circ} \pm 2^{\circ}$ in both samples of bulk NiO. Not only are the results consistent between the 
two samples, but the results of the experiments at the two instruments DMC and D7 also agree. For the nanoparticle samples we also consistently find a significant out-of-plane spin angle. Depending on the choice of normalization to either the $\left\{\frac{3}{2} \frac{1}{2} \frac{1}{2}\right\}$ or the $\left\{\frac{3}{2} \frac{3}{2} \frac{1}{2}\right\}$ reflection, there is some inconsistency between the exact value of the determined spin angle. This is probably because of systematic errors in fitting the weak and broad $\left\{\frac{3}{2} \frac{3}{2} \frac{1}{2}\right\}$ peak for the small particles. Most significantly, the $\left\{\frac{3}{2} \frac{3}{2} \frac{3}{2}\right\}$ peak at $q=3.9 \AA^{-1}$ contributes somewhat to the intensity of $\left\{\frac{3}{2} \frac{3}{2} \frac{1}{2}\right\}$, leading to a smaller value of $\alpha$. For this reason we will treat the results obtained from the relative intensities of $\left\{\frac{1}{2} \frac{1}{2} \frac{1}{2}\right\}$ and $\left\{\frac{3}{2} \frac{1}{2} \frac{1}{2}\right\}$ as our best estimate of $\alpha$ and not discuss the results obtained using the $\left\{\frac{3}{2} \frac{3}{2} \frac{1}{2}\right\}$ intensity.

For the nanoparticle samples with particle sizes larger than $2 \mathrm{~nm}(\mathrm{NiO} 300, \mathrm{NiO} 350$, and $\mathrm{NiO} 600)$ we find out-of-plane spin angles similar to that found in the two bulk samples ( $16^{\circ}$ on average). In the $\mathrm{NiO} 250$ sample, which consists of particles only $2.0 \mathrm{~nm}$ thick, we find a larger out-of-plane angle of $30^{\circ}$

It may be noted here that the measured (normalized) cross section is proportional to $\sin ^{2} \alpha$ [see Eq (9)] and that the sine of an angle changes very little with variation of the angle, when the angle is close to $90^{\circ}$ [for example, $\sin \left(74^{\circ}\right)=0.96 \approx 1$ ]. This means that, in determining $\alpha$, we are susceptible to even rather small systematic errors. Therefore, the value of $\alpha$ should perhaps not be taken as a precise number for the samples with $\sin \alpha \approx 1$ (i.e., all but $\mathrm{NiO} 250$ ). For the $\mathrm{NiO} 250$ sample the value of $\alpha=60^{\circ}$ deviates more significantly from $90^{\circ}$ and is thus more reliable $\left(\sin 60^{\circ}=0.87\right)$.

It is possible that the exact spin configuration in $\mathrm{NiO}$ can be sample dependent. In the experiment of Ressouche et al. [11], where the spin is found to be within $1^{\circ}$ of the (111) plane, the sample is a single crystal of $\mathrm{NiO}$, which may not be directly comparable to our bulk $\mathrm{NiO}$ powder. Impurities could obviously have an effect on the spin structure. However, our bulk samples NiO-Alfa and NiO1100 both have a high purity. Furthermore, the two samples are produced in different ways, but have the same spin angle, and it is therefore highly unlikely that impurities can be responsible for the out-of-plane spin component in our samples. Crystallographic strain could have an influence on the spin structure because of the exchange-striction coupling between the spin and crystallographic structure [22] and this could possibly explain the difference between the single crystal of Ressouche et al. and our powder samples.

We cannot rule out that some systematic error in our experiments could explain the (small) deviation from $90^{\circ}$ in the spin angle for all but the $\mathrm{NiO} 250$ sample. However, it should be noted that the experiments on two different instruments (DMC and D7) results in the same spin angle. One might be concerned that preferred orientation of the powder could be a problem for the platelet-shaped particles, but the TEM images and the similarity between the count rates in the three orthogonal channels in the D7 experiments show that there is no pronounced tendency to preferred orientation. Furthermore, the bulk samples and in particular the NiO-Alfa sample, which does not consist of platelet-shaped particles, would not be expected to show any particular degree of preferred orientation.
From the unpolarized experiment it was possible to determine the size of the $\mathrm{Ni}^{2+}$ magnetic moment in the $\mathrm{NiO} 600$ sample and the two bulk samples from the intensity of the $\left\{\frac{1}{2} \frac{1}{2} \frac{1}{2}\right\}$ peak normalized to one of the nuclear peaks. The measured moments for the two bulk samples $\left(\mu=2.01 \mu_{B} \pm\right.$ $0.04 \mu_{B}$ and $\left.\mu=2.02 \mu_{B} \pm 0.04 \mu_{B}\right)$ and the NiO600 sample $\left(\mu=2.19 \mu_{B} \pm 0.07 \mu_{B}\right)$ agree well with literature values for bulk $\mathrm{NiO}\left(1.8 \mu_{B}-2.2 \mu_{B}\right)$ [23-26]. If there is a so-called magnetic dead layer near the surface of the particles, in which the spins are disordered, the measured magnetic moment will be underestimated. This is, however, not likely to be important for bulk samples, but for the NiO600 sample a significant fraction of the atoms reside near the surface (about $9 \%$ of the atoms are within one lattice constant of the surface if a spherical particles shape is assumed) and the effect of a dead layer could be significant. By scaling the magnetic intensities in the polarized data to the magnetic moment of the NiO-Alfa bulk sample, the magnetic moments of the nanoparticle samples were determined to $2.20 \mu_{B} \pm 0.05 \mu_{B}$, $1.98 \mu_{B} \pm 0.06 \mu_{B}, 2.08 \mu_{B} \pm 0.05 \mu_{B}$, and $1.97 \mu_{B} \pm 0.08 \mu_{B}$ for $\mathrm{NiO} 600, \mathrm{NiO} 350, \mathrm{NiO} 300$, and $\mathrm{NiO} 250$, respectively. For $\mathrm{NiO} 600$ the result is in excellent agreement with the result from the unpolarized experiment at DMC. For the samples with smaller particle sizes the moments are the same as for the bulk sample within the measurement uncertainty. The fact that the size of the magnetic moment does not change from bulk to the smallest particles suggests that the magnetic order continues throughout the volume of the particles. The reason for the slightly larger magnetic moment in NiO600 is not clear.

In a powder diffraction experiment the measured quantities are averaged over the entire ensemble, i.e., over all particles in the sample and over all atoms in each particle. This means that we cannot distinguish whether the measured outof-plane spin angle corresponds to pronounced spin canting at the surface of the particles due to the low-symmetry surroundings of surface spins or a rotation of the spins in the entire particle due to a significant surface contribution to the magnetic anisotropy. Both localized canting at the particle surfaces and rotation of the entire sublattices could explain a spin rotation away from the (111) planes in the smallest particles (NiO250). Measurements of exchange bias and large coercivities in $\mathrm{NiO}$ nanoparticles, however, have been interpreted as evidence for interaction between the antiferromagnetic particle core and canted spins at the particle surfaces [28,33-36,44]. Furthermore, the measurements of Cooper et al. [19] on 7-nm spherical $\mathrm{NiO}$ nanoparticles confirmed that there is a disordered shell of spins that do not follow the antiferromagnetic order of the particle core and this suggests that our measured out-of-plane spin angle most likely has its origin at the surface of the particles.

\section{CONCLUSION}

We have studied the spin orientation in bulk $\mathrm{NiO}$ and in platelet-shaped nanoparticles of $\mathrm{NiO}$ with thickness down to $2.0 \mathrm{~nm}$ with unpolarized and polarized neutron powder diffraction. We found that the spin angle is close to the (111) plane $\left(\approx 16^{\circ}\right.$ out of plane $)$ in platelet-shaped $\mathrm{NiO}$ particles with thickness down to $2.2 \mathrm{~nm}$ and in bulk NiO powder. For plateletshaped $\mathrm{NiO}$ particles with a platelet thickness of only $2.0 \mathrm{~nm}$, 
we found that the spins are tilted as much as $30^{\circ}$ out of the (111) plane of the particles. The out-of-plane spin angle could be due to localized spin canting at the surface of the particles.

We found that the size of the magnetic moment in plateshaped $\mathrm{NiO}$ nanoparticles with thickness between 2.0 and 3.3 $\mathrm{nm}$ is the same as in the bulk samples, suggesting that there is no extended magnetic dead layer near the particle surfaces. Our experiments have shown that $X Y Z$-polarization analysis can be successfully used to study the magnetic structure of antiferromagnetic nanoparticles, obtaining results that would not have been possible without polarization analysis, and the $X Y Z$-polarization method can thus be very useful for future investigations of magnetic structures of antiferromagnetic nanoparticles.

\section{ACKNOWLEDGMENTS}

The experimental work was performed at ILL, Grenoble, and SINQ, PSI, Villigen. The project was funded by the Danish Council for Independent Research through DanScatt.
[1] J. Nogués, J. Sort, V. Langlais, V. Skumryev, S. Suriñach, J. Muñoz, and M. Baró, Phys. Rep. 422, 65 (2005).

[2] V. Skumryev, S. Stoyanov, Y. Zhang, G. Hadjipanayis, D. Givord, and J. Nogués, Nature (London) 423, 850 (2003).

[3] J.-H. Lee, J.-T. Jang, J.-S. Choi, S. H. Moon, S.-H. Noh, J.-W. Kim, J.-G. Kim, I.-S. Kim, K. I. Park, and J. Cheon, Nat. Nanotechnol. 6, 418 (2011).

[4] C. G. Shull, W. A. Strauser, and E. O. Wollan, Phys. Rev. 83, 333 (1951).

[5] L. Néel, Compt. Rend. 203, 304 (1936).

[6] J. R. Tomlinson, L. Domash, R. G. Hay, and C. W. Montgomery, J. Am. Chem. Soc. 77, 909 (1955).

[7] W. L. Roth, Phys. Rev. 110, 1333 (1958).

[8] W. L. Roth and G. A. Slack, J. Appl. Phys. 31, S352 (1960).

[9] M. T. Hutchings and E. J. Samuelsen, Phys. Rev. B 6, 3447 (1972).

[10] J. Baruchel, M. Schenker, K. Kurosawa, and S. Saito, Philos. Mag. B 43, 853 (1981).

[11] E. Ressouche, N. Kernavanois, L.-P. Regnault, and J.-Y. Henry, Physica B 385-386, 394 (2006).

[12] L. Berger, Y. Labaye, M. Tamine, and J. M. D. Coey, Phys. Rev. B 77, 104431 (2008).

[13] R. H. Kodama, J. Magn. Magn. Mater. 200, 359 (1999).

[14] J. Li, E. Arenholz, Y. Meng, A. Tan, J. Park, E. Jin, H. Son, J. Wu, C. A. Jenkins, A. Scholl, H. W. Zhao, C. Hwang, and Z. Q. Qiu, Phys. Rev. B 84, 012406 (2011).

[15] D. S. Deng, X. F. Jin, and R. Tao, Phys. Rev. B 69, 172403 (2004).

[16] G. L. Squires, Thermal Neutron Scattering (Cambridge University Press, Cambridge, 1978).

[17] S. N. Klausen, P.-A. Lindgård, K. Lefmann, F. Bødker, and S. Mørup, Physica Status Solidi A 189, 1039 (2002).

[18] C. Frandsen, K. Lefmann, B. Lebech, C. R. H. Bahl, E. Brok, S. N. Ancoña, L. Theil Kuhn, L. Keller, T. Kasama, L. C. Gontard, and S. Mørup, Phys. Rev. B 84, 214435 (2011).

[19] J. F. K. Cooper, A. Ionescu, R. M. Langford, K. R. A. Ziebeck, C. H. W. Barnes, R. Gruar, C. Tighe, J. A. Darr, N. T. K. Thanh, and B. Ouladdiaf, J. Appl. Phys. 114, 083906 (2013).

[20] O. Schärpf and H. Capellmann, Physica Status Solidi A 135, 359 (1993).

[21] F. Fiévet, P. Germi, F. de Bergevin, and M. Figlarz, J. Appl. Crystallogr. 12, 387 (1979).
[22] L. C. Bartel and B. Morosin, Phys. Rev. B 3, 1039 (1971).

[23] W. Neubeck, C. Vettier, V. Fernandez, F. de Bergevin, and C. Giles, J. Appl. Phys. 85, 4847 (1999).

[24] V. Fernandez, C. Vettier, F. de Bergevin, C. Giles, and W. Neubeck, Phys. Rev. B 57, 7870 (1998).

[25] S. K. Kwon and B. I. Min, Phys. Rev. B 62, 73 (2000).

[26] B. E. F. Fender, A. J. Jacobson, and F. A. Wedgwood, J. Chem. Phys. 48, 990 (1968).

[27] J. T. Richardson and W. O. Milligan, Phys. Rev. 102, 1289 (1956).

[28] S. A. Makhlouf, F. T. Parker, F. E. Spada, and A. E. Berkowitz, J. Appl. Phys. 81, 5561 (1997).

[29] F. Bødker, M. F. Hansen, C. B. Koch, and S. Mørup, J. Magn. Magn. Mater. 221, 32 (2000).

[30] Y. Ichiyanagi, N. Wakabayashi, J. Yamazaki, S. Yamada, Y. Kimishima, E. Komatsu, and H. Tajima, Physica B 329-333, 862 (2003).

[31] C. R. H. Bahl, M. F. Hansen, T. Pedersen, S. Saadi, K. H. Nielsen, B. Lebech, and S. Mørup, J. Phys.: Condens. Matter 18, 4161 (2006).

[32] C. R. H. Bahl, The Magnetic Properties of Antiferromagnetic Nanoparticles: $\mathrm{NiO}$ and $\alpha-\mathrm{Fe}_{2} \mathrm{O}_{3}, \mathrm{Ph} . \mathrm{D}$. thesis, Ris $\varnothing$ National Laboratory, 2006.

[33] E. Winkler, R. D. Zysler, M. Vasquez Mansilla, and D. Fiorani, Phys. Rev. B 72, 132409 (2005).

[34] J. B. Yi, J. Ding, Y. P. Feng, G. W. Peng, G. M. Chow, Y. Kawazoe, B. H. Liu, J. H. Yin, and S. Thongmee, Phys. Rev. B 76, 224402 (2007)

[35] E. Winkler, R. D. Zysler, M. Vasquez Mansilla, D. Fiorani, D. Rinaldi, M. Vasilakaki, and K. N. Trohidou, Nanotechnology 19, 185702 (2008)

[36] M. Jagodič, Z. Jagličić, A. Jelen, J. B. Lee, Y.-M. Kim, H. J. Kim, and J. Dolinšek, J. Phys.: Condens. Matter 21, 215302 (2009).

[37] C. R. H. Bahl, K. Lefmann, L. Theil Kuhn, N. B. Christensen, H. Vázquez, and S. Mørup, J. Phys.: Condens. Matter 18, 11203 (2006).

[38] J. T. Richardson, D. I. Yiagas, B. Turk, K. Forster, and M. V. Twigg, J. Appl. Phys. 70, 6977 (1991).

[39] R. H. Kodama, S. A. Makhlouf, and A. E. Berkowitz, Phys. Rev. Lett. 79, 1393 (1997).

[40] A. E. Berkowitz, R. H. Kodama, S. A. Makhlouf, F. T. Parker, F. E. Spada, E. J. McNiff, Jr., and S. Foner, J. Magn. Magn. Mater. 196-197, 591 (1999). 
[41] V. V. Pishko, S. L. Gnatchenko, V. V. Tsapenko, R. H. Kodama, and S. A. Makhlouf, J. Appl. Phys. 93, 7382 (2003).

[42] J. R. Stewart, P. P. Deen, K. H. Andersen, H. Schober, J.-

F. Barthélémy, J. M. Hillier, A. P. Murani, T. Hayes, and B. Lindenau, J. Appl. Crystallogr. 42, 69 (2008).
[43] C. Frandsen, C. R. H. Bahl, B. Lebech, K. Lefmann, L. Theil Kuhn, L. Keller, N. H. Andersen, M. v. Zimmermann, E. Johnson, S. N. Klausen, and S. Mørup, Phys. Rev. B 72, 214406 (2005).

[44] S. A. Makhlouf, H. Al-Attar, and R. Kodama, Solid State Commun. 145, 1 (2008). 